\title{
Assessment of the burden of diseases and injuries attributable to risk factors in Canada from 1990 to 2016: an analysis of the Global Burden of Disease Study
}

\author{
Samiah Alam MSc, Justin J. Lang PhD, Aaron M. Drucker MD ScM, Carolyn Gotay PhD, \\ Nicole Kozloff MD SM, Kedar Mate MSc, Scott B. Patten MD PhD, Heather M. Orpana PhD, \\ Ashkan Afshin MD ScD, Leah E. Cahill PhD
}

\section{Abstract}

Background: An understanding of the risk factors contributing to disease burden is critical for determining research priorities and informing national health policy. We aimed to identify the risk factor trends in Canada.

Methods: As part of the Global Burden of Disease (GBD) study (1990-2016), we conducted an analysis of country-level estimates for Canada to assess the burden of diseases and injuries attributable to risk factors. For both 1990 and 2016, metabolic, environmental and behavioural risk factors were ranked according to their contribution to disability-adjusted life years (healthy years of life lost), total deaths and years lived with disability.

Results: In 2016, the risk factors accounting for the largest percentage of disability-adjusted life years in Canada were (1) tobacco, (2) diet, (3) high body mass index, (4) high fasting plasma glucose, (5) high systolic blood pressure, (6) alcohol and drug use, (7) occupational risks, (8) high total cholesterol, (9) impaired kidney function and (10) air pollution. Risk factor rankings remained similar from 1990 to 2016 despite some substantial declines in burden, including a $47 \%( \pm 3 \%)$ decline in the age-standardized disabilityadjusted life years rate attributable to tobacco since 1990. Risk factors with an increasing contribution to disability-adjusted life years rates from 1990 to 2016 included high body mass index, high fasting plasma glucose and alcohol and drug use.

Interpretation: Metabolic and behavioural risk factors, including modifiable factors such as tobacco use and diet, remain the leading risk factors contributing to the burden of diseases and injuries in Canada. This work identifies priorities and targets for reducing premature death and disability burden in Canada.

I n Canada, modifiable factors such as smoking, diet and physical activity are major determinants of life expectancy, ${ }^{1}$ indicating that actionable preventive strategies can lead to improved health outcomes. An understanding of Canada's foremost health problems, their risk factors, and how both are changing over time is crucial to inform national health policy and programs, health authority planning and scientific research priorities. However, disease burden has traditionally been defined in terms of mortality alone, which misses additional health burden, such as living with a disability. It is difficult for policy-makers and researchers to set priorities when disparate diseases and injuries affect population health in diverse ways, and the measurement of their contributions to health burden is not standardized. Therefore, an analysis that examines health loss from both disability and death on the same scale in relation to attributable risk factors is warranted.
The Global Burden of Diseases, Injuries, and Risk Factors Study (GBD) is an international scientific effort to systematically quantify the comparative magnitude of global health loss resulting from disease, injuries and risk factors to inform evidence-based policy-making. ${ }^{2}$ In the GBD, the goal is not just to measure which diseases or injuries cause death but also to measure which diseases and injuries prevent someone from

Competing interests: Aaron Drucker reports grants and personal fees from Sanofi and Regeneron for activities outside the work reported here. No other competing interests were declared.

This article has been peer reviewed.

Correspondence to: Leah Cahill, leah.cahill@dal.ca

CMAJ Open 2019. DOI:10.9778/cmajo.20180137 
living a productive, active and full life. ${ }^{3}$ GBD burden estimates for health loss and attributable risk factors have recently been summarized for individual countries, including the United States, ${ }^{4,5}$ Germany $^{6}$ and the United Kingdom. ${ }^{7}$ Although estimates of health loss have recently been presented for Canada, ${ }^{8}$ the burden of attributable risk factors for Canada has not yet been reported. The objective of this paper is to identify the leading risk factors contributing to the burden of diseases and injuries in Canada.

\section{Methods}

The GBD study, led by the Institute for Health Metrics and Evaluation at the University of Washington, quantifies global health loss to inform evidence-based policy-making. ${ }^{9}$ The GBD study has an established reputation for providing comprehensive assessments of global mortality and morbidity, and its comparative risk assessment approach to quantifying the influence of risk factors on disease burden is well regarded. The GBD study collects and analyzes a continuous stream of the most up-to-date data available from a wide variety of resources including randomized controlled trials, cohort studies, household surveys, census data, vital registration data and satellite data. ${ }^{10}$ These data are extracted into centralized databases to model internally consistent estimates for diseases, injuries and risk factors, adjusting for bias, standardizing for age and incorporating covariates. ${ }^{10}$ Specific details of the GBD study methods and their development (including analytic tools developed for missing data) have been reported previously, ${ }^{11-16}$ and GBD methods, data and codes are available publicly through interactive tools and visualizations (vizhub.healthdata.org/gbd-compare). ${ }^{17}$ The GBD methodology is reviewed and updated annually and is compliant with the Guidelines for Accurate and Transparent Health Estimates Reporting (GATHER). ${ }^{18}$

Members of the author group for the present paper are GBD study collaborators. To identify Canadian trends in risk factors for the present paper, we conducted an analysis of GBD country-level Canadian data from 1990 (the first year of the GBD study) to 2016 (the most recent year).

\section{Data sources}

Canadian estimates for the present paper were synthesized from over 600 data sources, of which 141 were used for the risk factors assessment (ghdx.healthdata.org/gbd-2016/ data-input-sources).

\section{Measurement of burden}

Three well-established metrics summarize health loss: ${ }^{12,14}$ (a) summation of premature death and disability (disabilityadjusted life years [DALYs]), (b) death (total deaths) and (c) disability (years lived with disability ${ }^{19-21}$ ). One DALY is equivalent to 1 year of healthy life (free of disease, injury or disability) lost. ${ }^{12}$ Because DALYs quantify both mortality and morbidity, total DALYs are considered to represent the burden of diseases and injuries for a country, indicating the gap between the country's current health status and an ideal health situation in which the country's entire population lives to an advanced age, free of disease and disability. ${ }^{5}$

\section{Selection of risk factors}

In the GBD study, a risk is an attribute, behaviour, exposure or other factor causally associated with an increased probability of developing a disease or experiencing an injury. A total of 481 risk-outcome pairs were identified as meeting the evidence grading criteria of being biologically plausible and showing consistent associations between exposure and disease, with little or no evidence to the contrary. ${ }^{10}$ Our comparative risk assessment conceptual framework employs a causal web in which the risks that are responsible for the health outcomes are organized into 4 hierarchical, mutually exclusive and collectively exhaustive levels. ${ }^{10,22}$ Level 1 includes 3 categories of risk factors (behavioural, environmental and occupational, and metabolic) that distribute into 17 level 2 risk factors as either single risks or risk clusters (Appendix 1, Supplementary Table 1, available at www.cmajopen.ca/content/7/1/E140/ suppl/DC1). While not within the scope of this paper, levels 3 and 4 have 50 and 67 risk factors, respectively.

\section{Attributable burden estimation and statistical analysis}

The calculation of attributable burden for a risk factor has 7 steps and 4 components (Figure 1): exposure levels for the risk, the relative risk of a health outcome related to the risk exposure, a counterfactual (optimal) level of risk factor exposure, and the burden metric (DALYs, number of deaths or years lived with disability). ${ }^{10}$ The theoretical minimum risk exposure level for a risk factor represents the optimal exposure level that minimizes risk for everyone in the population. These input data are used to estimate the population attributable fraction, which is the proportion of the disease burden that could have been prevented if the risk factor was at the theoretical minimum risk exposure level. The population attributable fraction is then multiplied by the disease-specific burden metric to obtain attributable disease burden for each risk-outcome pair for each time point, accompanied by $95 \%$ uncertainty intervals (UI) that incorporate both sampling error and model estimation error. Risk factors were ranked from high to low on the rate (per 100 000) of DALYs, deaths and years lived with disability they contributed to for each time point. To explain the drivers of our observed trends in Canada's risk-attributable disease and injury burden, we conducted a decomposition analysis using methods ${ }^{15}$ based on those of Das Gupta ${ }^{23}$ to examine the percentage change in Canada's risk-attributable DALYs over time resulting from (a) population growth, (2) population aging, (c) risk-deleted mortality rates (the expected mortality that would be observed if all risk factors included in GBD $2016^{15}$ were removed) and (d) exposure to all risk factors included in GBD 2016.

\section{Ethics approval}

Ethics approval was not sought because the GBD study is an analysis of multiple previous data sources for which ethics approvals were maintained individually. 


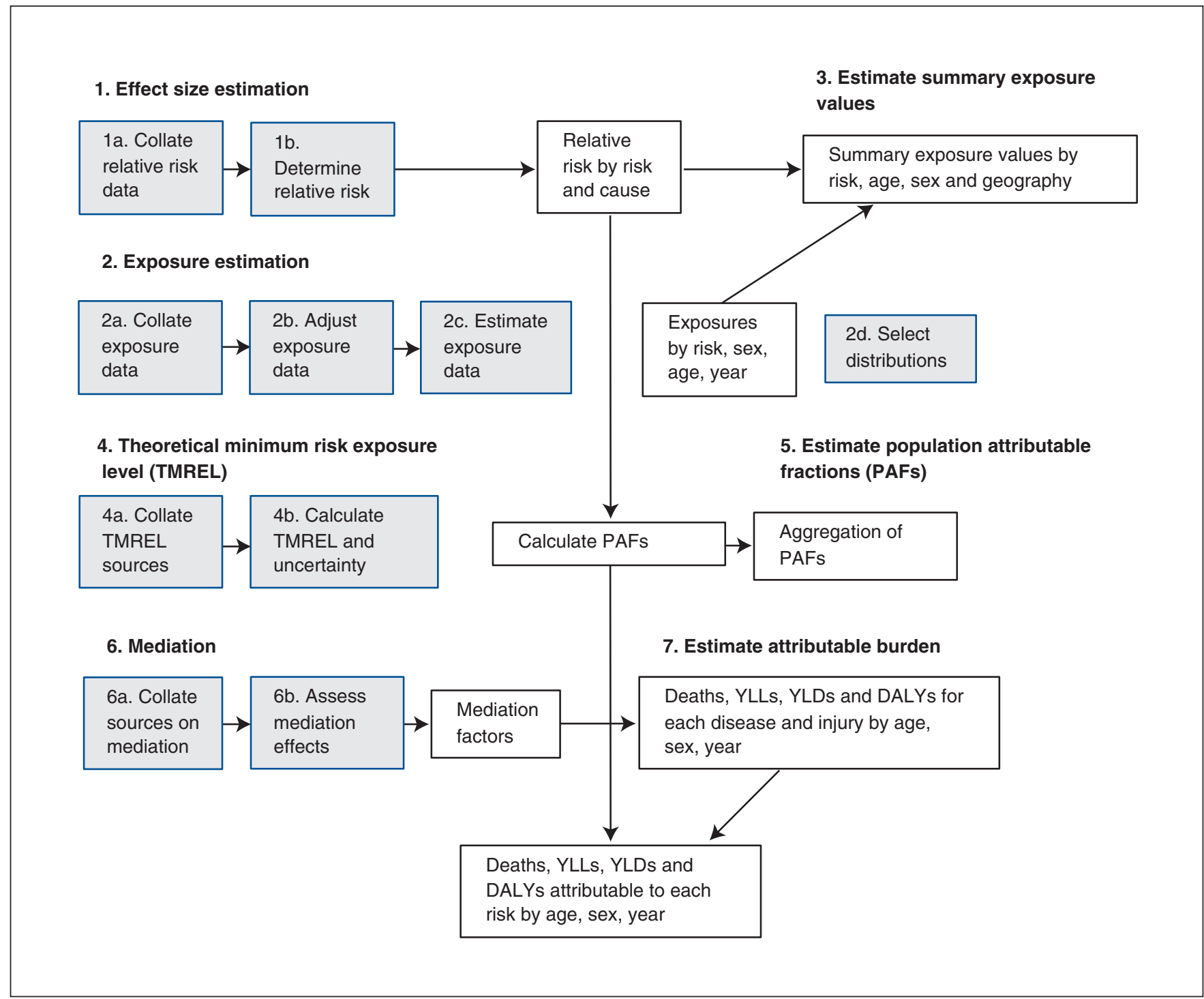

Figure 1: Simplified analytical flowchart outlining the 7 steps of the comparative risk assessment for the estimation of population attributable fractions by geography, age, sex and year. Note: DALYs = disability-adjusted life years, PAFs = population attributable fractions, TMREL = theoretical minimum risk exposure level, YLDs = years lived with disability, YLLs = years of life lost.

\section{Results}

In analyzing level 0 risk factor (all-age and all-cause) GBD study data, we estimated that $39.6 \%(36.9 \%$ to $42.3 \%)$ of DALYs, $56.0 \%$ (53.6\% to $58.2 \%)$ of deaths and $24.2 \%$ (22.7\% to $25.8 \%)$ of years lived with disability in Canada in 2016 could be attributed to the assessed risk factors.

\section{Top 10 risk factors for attributable DALYs}

In 2016, the 10 leading risk factors contributing to all-cause DALYs in Canada (reported as \% of total DALYs [95\% UT]) were tobacco (10.6\% [9.4 to 11.9$]$ ), dietary risks $(9.4 \%$ [7.7\% to $11.2 \%])$, high body mass index (BMI) $(9.0 \%$ [6.2\% to $11.8 \%])$, high fasting plasma glucose (7.3\% [5.8\% to $9.2 \%])$, high systolic blood pressure (7.1\% [6.0\% to $8.2 \%]$ ), alcohol and drug use $(6.9 \%$ [5.8\% to $8.1 \%])$, occupational risks (4.8\% [4.4\% to
$5.3 \%]$ ), high total cholesterol (3.7\% [2.9\% to 4.6\%]), impaired kidney function (2.1\% [1.9\% to $2.3 \%]$ ) and air pollution $(1.3 \%$ [1.0\% to $1.7 \%]$ ), mainly through their impact on cancer and cardiovascular disease (Figure 2, Appendix 1, Supplementary Table 2). The leading risk factors for DALYs were similar for men and women, except for alcohol and drug use (ranked higher for men) and high systolic blood pressure (ranked higher for women) (Appendix 1, Supplementary Figure 1). Stratification by age group also revealed differences in risk factor attribution (Appendix 1, Supplementary Figures 7 and 8).

\section{Top 5 risk factors for attributable deaths and years lived with disability}

In terms of total deaths in 2016, the 5 leading all-cause, allage attributable risk factors (reported as \% of total deaths $[95 \%$ UI] $)$ were dietary risks $(17.6 \%[14.7 \%$ to $20.5 \%]$ ), 
tobacco $(17.5 \%$ [ $16.6 \%$ to $18.5 \%])$, high systolic blood pressure $(14.7 \%$ [12.3\% to $17.2 \%]$ ), high fasting plasma glucose (11.2\% [7.9\% to $15.3 \%]$ ) and high BMI (10.8\% [6.9\% to 15.0\%]) (Figure 2B, Appendix 1, Supplementary Table 2). High BMI was the top risk factor contributing to years lived with disability in Canada (7.3\% [5.1\% to $9.4 \%]$ ), followed by high fasting plasma glucose $(5.5 \%$ [4.8\% to $6.3 \%])$ and alcohol and drug use (5.4\% [4.5\% to 6.5\%]) (Figure 2C). Cancer and heart disease were the causes through which risk factors imparted most of their impact on total deaths, while diabetes and musculoskeletal disorders were the top causes through which risk factors influenced years lived with disability.

\section{Trends from 1990 to 2016 for DALYs, deaths and years lived with disability}

Since 1990, exposure to tobacco has continued to be the leading risk factor for all-cause DALYs even though declines in the tobacco-attributable all-age DALY rate $(-25.7 \%$ [95\% UI $-30.1 \%$ to $-21.1 \%]$ ) and the tobacco-attributable age-standardized DALY rate $(-47.4 \%$ [ $-50.5 \%$ to $-44.1 \%)]$ were observed over the last 27 years (Figure 3A). High BMI and high fasting plasma glucose have both increased in ranking to become the third and fourth leading risk factors. Alcohol and drug use attributable all-age and age-standardized DALY rates have increased by $13.3 \%(0.0 \%$ to $30.4 \%)$ and $8.5 \%$ ( $-5.4 \%$ to $26.4 \%)$ since 1990 . The all-age attributable DALY rate has decreased for high systolic blood pressure $(-34.4 \%$ [ $-38.6 \%$ to $-30.0 \%])$ and high cholesterol $(44.5 \%$ [-48.8\% to $-39.8 \%]$ ) since 1990 , leading to improvements in their respective rankings.

Among the leading risk factors contributing to all-cause total deaths, the risk factor contributing to a substantial increase in the death rate from 1990 to 2016 was high BMI, which increased by $22.3 \%$ ( $4.2 \%$ to $54.8 \%$ ) (Figure 3B). Alcohol and drug use ranked ninth as an attributable risk for deaths, but its contribution to the all-age and age-standardized death rates has risen by $66.4 \%$ (13.6\% to $251.1 \%$ ) and $36.4 \%$ (-7.8\% to $202.2 \%)$, respectively, since 1990 . The risk factors contributing to all-cause years lived with disability have changed from 1990, with high BMI and high fasting plasma glucose becoming the top 2 risk factors, reflecting a $61.0 \%$ ( $46.0 \%$ to $85.8 \%$ ) and $40.2 \%$ (26.5\% to $55.3 \%)$ increase in the all-age attributable years lived with disability rates associated with these risk factors (Figure 3C).

\section{Drivers of observed trends in risk-attributable DALYs}

Our decomposition analysis separated the relative contributions of risk factors from those of population growth, aging and risk-deleted mortality rate, revealing that communicable and noncommunicable diseases attributable to risk factor exposure are declining in Canada, while the percentage of injuries resulting from risk factor exposure is increasing (Figure 4). For all ages combined, exposure to risk factors is accountable for a decreasing percentage of health burden in Canada. However, stratification by age group reveals that exposure to risk factors accounts for an increasing percentage of health burden in Canadians aged 5-9 and 20-39 years.

\section{Interpretation}

Of the risk factors included in our GBD study estimates, the top 2 level 2 risk factors related to health burden (premature death and disability combined) in Canada are tobacco and diet, mainly through their impact on cancer and cardiovascular disease. This ranking has remained relatively unchanged since 1990, despite substantial declines in burden owing to diet and tobacco. High systolic blood pressure and high total cholesterol have decreased in ranking, but they remain important risk factors. The contribution of alcohol and drug use, high BMI and high fasting plasma glucose to the burden of diseases and injuries increased from 1990 to 2016. The 2 leading risk factors for disability were high BMI and high fasting plasma glucose (through endocrine and musculoskeletal disorders) and the 2 leading risk factors for mortality were diet and tobacco (through cancer and cardiovascular disease).

Although tobacco exposure is still a leading risk factor, its contribution to DALYs was much lower in Canadians younger than 49 years than in older Canadians and it declined considerably over the study period, which is consistent with the long latency period of lung cancer and other tobaccorelated diseases, historical patterns of tobacco use and Canadian smoking cessation programs. ${ }^{24-26}$ Dietary risk has remained the second largest contributor to health burden in Canada over the last quarter century, probably because of the involvement of multiple aspects of nutrition (changes in the amount and sources of calories, the accessibility of foods and the intake of different nutrients) in the pathophysiology of many diseases and multiple intermediary risk factors including high BMI and high fasting plasma glucose. ${ }^{15,17}$

Few previous studies have undertaken the task of identifying the top attributable risk factors for disease burden in Canada, and those that did focused on mortality as an outcome. The results of the present study are comparable with a study that used the Mortality Population Risk Tool (MPoRT); the authors reported that $50 \%$ of deaths in Canada could be attributed to the 4 behavioural risk factors they assessed (smoking, unhealthy alcohol consumption, physical inactivity and poor diet). ${ }^{1}$ Similar to our results, smoking was the leading behaviour contributing to deaths for men, despite a continuing decrease in Canadian smoking rates. ${ }^{1}$ Steensma and colleagues also demonstrated significant health loss in the Canadian population associated with high BMI. ${ }^{27}$ A 2006 analysis reported that alcohol is a major contributor to mortality in Canada, ${ }^{28}$ as we also observed in the present study, and as was reported in a previous (2005) GBD analysis of the premature mortality attributable to alcohol consumption in Canada. ${ }^{29}$ Internationally, recent analyses of US ${ }^{4}$ and $\mathrm{UK}^{7}$ GBD data have reported similar results to ours, with tobacco and diet as the 2 leading risk factors contributing to health burden.

\section{Limitations}

The present analysis used Canadian data from numerous sources across the country; however, important sources of data may not yet be included in the GBD study. With current GBD Canada estimates we are unable to explain why 
A

Tobacco
Dietary risks
High body mass index
High fasting plasma glucose
High systolic blood pressure
Alcohol and drug use
Occupational risks
High total cholestero
Impaired kidney function
Air pollution
Low physical activity
Child and maternal malnutrition
Low bone mineral density
Unsafe sex
Sexual abuse and violence
Other environmental risks

Unsafe water, sanitation and handwashing

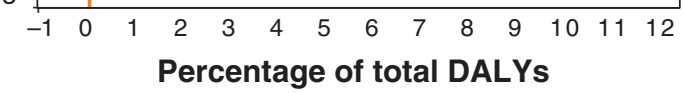

B

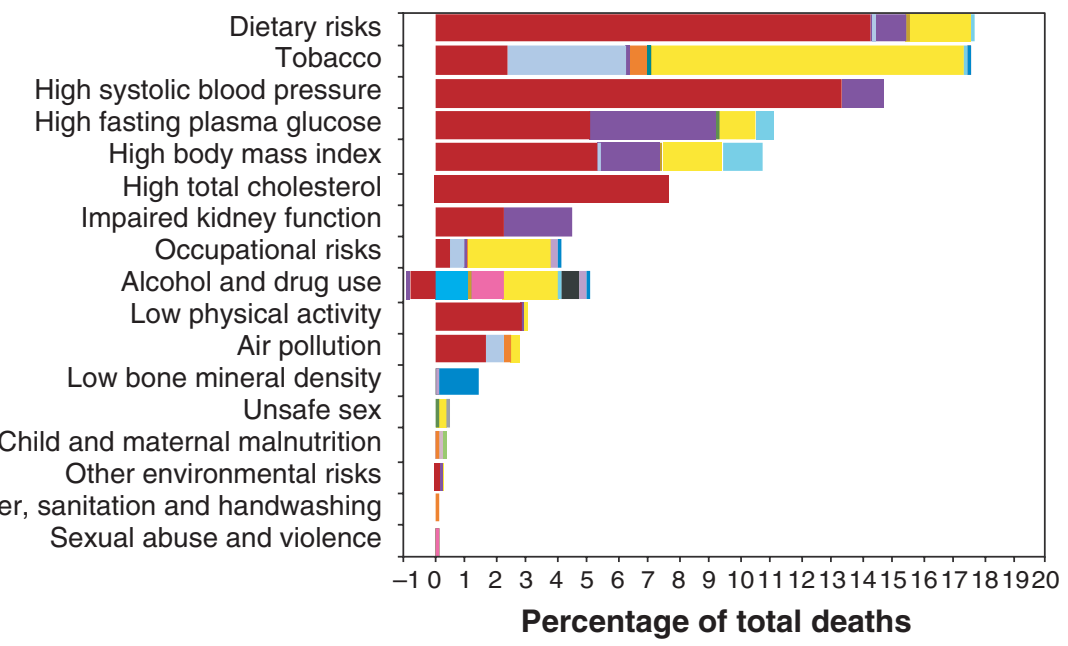

C

High body mass index High fasting plasma glucose Alcohol and drug use Occupational risks Dietary risks Tobacco

High systolic blood pressure Impaired kidney function Low bone mineral density

High total cholesterol

Sexual abuse and violence

Low physical activity Child and maternal malnutrition Unsafe sex

Other environmental risks Air pollution Unsafe water, sanitation and handwashing
Cardiovascular diseases

Chronic respiratory diseases

Cirrhosis and other chronic liver deases

Diabetes, urogenital, blood, and endocrine diseases

Digestive diseases

Mental and substance use disorders

- Musculoskeletal disorders

Neoplasms

Neurological disorders

Other noncommunicable diseases

- Self-harm and interpersonal violence

Transport injuries

- Unintentional injuries

War and disaster

Diarrhea, lower respiratory and other infectious diseases

- HIV/AIDS and tuberculosis

Maternal disorders

Nutritional deficiencies

Neonatal disorders

Other communicable, maternal, neonatal and nutritional diseases

Figure 2: Burden of disease attributable to leading risk factors for all Canadians in 2016 expressed as a percentage of (A) disability-adjusted life years (DALYs), (B) deaths and C) years lived with disability (YLDs). One DALY is equivalent to 1 year of healthy life (free of disease, injury or disability) that has been lost. The negative percentage for alcohol is the protective effect of mild alcohol use on risk of cardiometabolic disease. 
A

\begin{tabular}{l} 
Risk factors by attributable \\
DALYs 1990 \\
\begin{tabular}{|l|} 
1. Tobacco \\
DALk factors by attributable 2016
\end{tabular} \\
\hline 2. Dietary risks \\
\hline 3. High systolic blood pressure \\
\hline 4. High body mass index \\
\hline 5. High fasting plasma glucose \\
\hline 6. High total cholesterol \\
\hline 7. Alcohol and drug use \\
\hline 8. Occupational risks \\
\hline 9. Impaired kidney function \\
\hline 10. Air pollution \\
\hline \hline 11. Low physical activity \\
\hline 12. Child and maternal malnutrition \\
\hline 13. Unsafe sex \\
\hline 14. Low bone mineral density \\
\hline 15. Sexual abuse and violence \\
\hline 16. Other environmental risks \\
\hline 11. High \\
\hline $\begin{array}{l}\text { 17. Unsafe water, sanitation } \\
\text { and handwashing }\end{array}$ \\
\hline
\end{tabular}

$\%$ change \% change

all-age age-standarized DALY rate DALY rate
$(1990-2016)(1990-2016)$

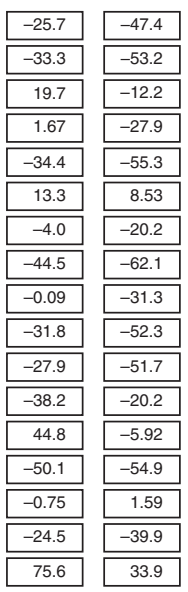

\section{C}

\begin{tabular}{|c|c|c|c|}
\hline $\begin{array}{c}\text { Risk factors by attributable } \\
\text { YLDs } 1990\end{array}$ & $\begin{array}{l}\text { Risk factors by attributable } \\
\text { YLDs } 2016\end{array}$ & $\begin{array}{l}\% \text { change } \\
\text { all-age ao } \\
\text { YLD rate } \\
\text { (1990-2016) }\end{array}$ & $\begin{array}{l}\% \text { change } \\
\text { ge-standarizec } \\
\text { YLD rate } \\
\text { (1990-2016) }\end{array}$ \\
\hline 1. Alcohol and drug use & 1. High body mass index & 61.0 & 24.3 \\
\hline 2. High body mass index & 2. High fasting plasma glucose & 40.2 & 2.92 \\
\hline 3. High fasting plasma glucose & 3. Alcohol and drug use & 11.2 & 16.0 \\
\hline 4. Occupational risks & 4. Occupational risks & 16.3 & 3.97 \\
\hline 5. Tobacco & 5. Dietary risks & 14.7 & -15.4 \\
\hline 6. Dietary risks & 6. Tobacco & -17.8 & -37.4 \\
\hline 7. High systolic blood pressure & 7. High systolic blood pressure & 6.81 & -26.0 \\
\hline 8. Impaired kidney function & 8. Impaired kidney function & 39.3 & 1.90 \\
\hline 9. High total cholesterol & 9. Low bone mineral density & 45.2 & -1.93 \\
\hline 10. Low bone mineral density & 10. High total cholesterol & -1.01 & -31.1 \\
\hline 11. Sexual abuse and violence & 11. Sexual abuse and violence & 6.07 & 8.73 \\
\hline 12. Low physical activity & 12. Low physical activity & 29.0 & $\overline{-11.3}$ \\
\hline 13. Child and maternal malnutrition & 13. Unsafe sex & 31.2 & 24.6 \\
\hline 14. Unsafe sex & 14. Child and maternal malnutrition & -6.61 & 10.2 \\
\hline 15. Air pollution & 15. Other environmental risks & 62.9 & 49.0 \\
\hline 16. Other environmental risks & 16. Air pollution & 30.7 & -6.73 \\
\hline $\begin{array}{l}\text { 17. Unsafe water, sanitation and } \\
\text { handwashing }\end{array}$ & $\begin{array}{l}\text { 17. Unsafe water, sanitation and } \\
\text { handwashing }\end{array}$ & -19.7 & -15.2 \\
\hline
\end{tabular}

B

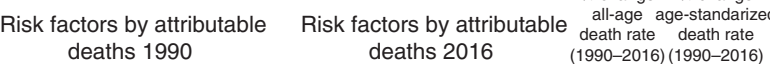

\begin{tabular}{|c|c|c|c|}
\hline 1. Dietary risks & 1. Dietary risks & -29.4 & -56.4 \\
\hline 2. Tobacco & 2. Tobacco & -14.3 & -43.3 \\
\hline 3. High systolic blood pressure & 3. High systolic blood pressure & -27.3 & -56.2 \\
\hline 4. High total cholesterol & 4. High fasting plasma glucose & 0.19 & -37.4 \\
\hline 5. High fasting plasma glucose & 5. High body mass index & 22.3 & -21.8 \\
\hline 6. High body mass index & 6. High total cholesterol & -36.1 & -61.8 \\
\hline 7. Occupational risks & 7. Impaired kidney function & 8.89 & -37.7 \\
\hline 8. Impaired kidney function & 8. Occupational risks & -1.07 & -28.4 \\
\hline 9. Low physical activity & 9. Alcohol and drug use & 66.4 & 36.4 \\
\hline 10. Air pollution & 10. Low physical activity & -19.2 & -52.6 \\
\hline 11. Alcohol and drug use & 11. Air pollution & -21.2 & -50.3 \\
\hline 12. Unsafe sex & 12. Low bone mineral density & 88.1 & 0.76 \\
\hline 13. Low bone mineral density & 13. Unsafe sex & -43.7 & -56.8 \\
\hline 14. Child and maternal malnutrition & 14. Child and maternal malnutrition & -41.7 & -23.2 \\
\hline 15. Other environmental risks & 15. Other environmental risks & -32.5 & -58.8 \\
\hline 16. Sexual abuse and violence & 16. Unsafe water, sanitation & 255 & 103 \\
\hline \multirow{2}{*}{$\begin{array}{l}\text { 17. Unsafe water, sanitation } \\
\text { and handwashing }\end{array}$} & & & \\
\hline & 17. Sexual abuse and violence & -14.5 & -21.2 \\
\hline
\end{tabular}

Behavioural risks

Metabolic risks

Environmental/occupational risks

Figure 3: (A) Rank changes in disability-adjusted life years (DALYs) attributable to leading risk factors and percentage change in all-age and age-standardized DALY rates in Canada from 1990 to 2016; 1 DALY is equivalent to 1 year of healthy life (free of disease, injury or disability) that has been lost. (B) Rank changes in total deaths attributable to leading risk factors and percentage change in all-age and age-standardized death rates in Canada between 1990 and 2016. (C) Rank changes in years lived with disability (YLDs) attributable to leading risk factors and percentage change in all-age and age-standardized years lived with disability rates in Canada between 1990 and 2016.

risk factors rose or fell in their rankings or to stratify by province/territory. A major limitation is that we could not incorporate data on the social determinants of health (e.g., housing, income, ethnicity, education) that strongly influence risk factors, ${ }^{30,31}$ differ vastly by community ${ }^{32,33}$ and are crucial to individual and population health. ${ }^{34-36}$ There may also be other risk factors that are important but not included in the
GBD, including psychosocial factors such as social isolation. ${ }^{37,38}$ Some diseases, such as depression and migraines, have a high burden and prevalence but little to no risk factor attributions in the present analysis. Additionally, estimates of the GBD study have not been validated against a similar study because no initiative of the same scope exists for comparison. The closest other study would be individual World 
A) DALYs, all age

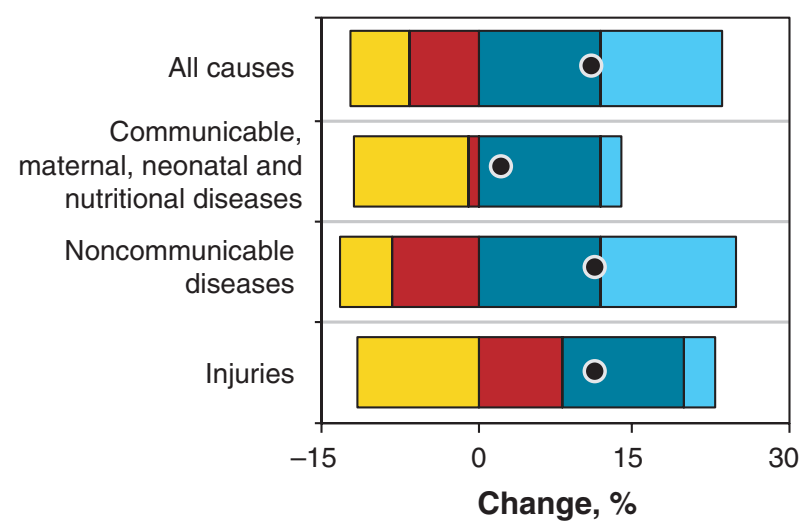

B) Deaths, all age

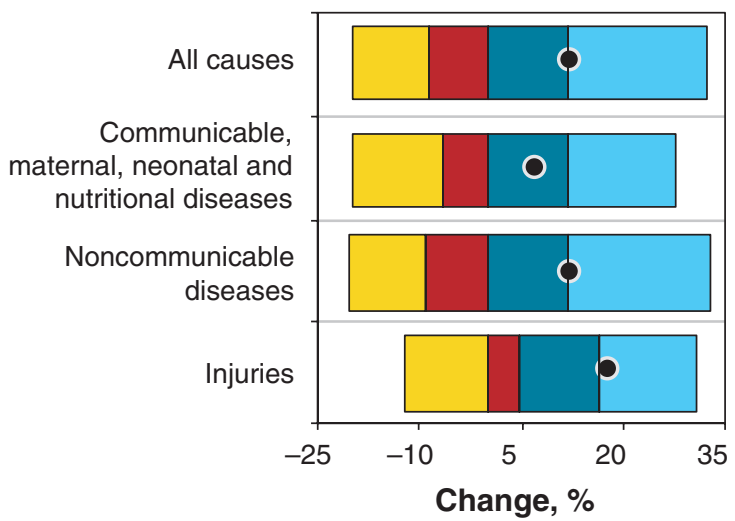

\section{C) DALYs, age stratified}

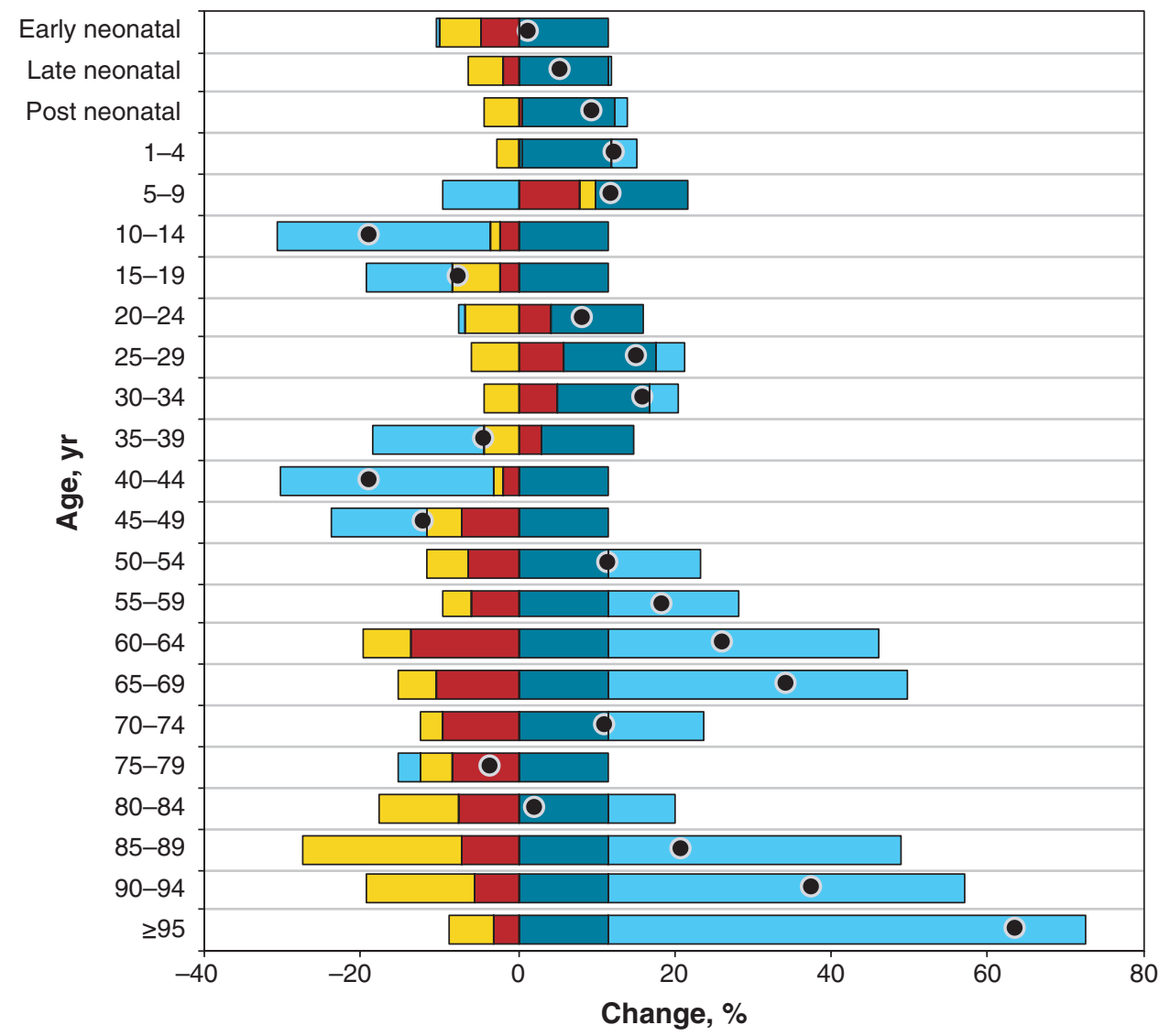

- Change due to risk exposure

$\square$ Change due to population aging

$\square$ Change due to risk-deleted mortality/DALY rate

- Total percentage change

$\square$ Change due to population growth

Figure 4: Drivers of percentage changes in Canada's risk-attributable disease and injury burden from 2006 to 2016 due to population growth, population aging, risk-deleted mortality and exposure to risk factors expressed as (A) disability-adjusted life years (DALYs) for all ages, (B) deaths for all ages and (C) DALYs by age group. One DALY is equivalent to 1 year of healthy life (free of disease, injury or disability) that has been lost. 
Health Organization (WHO) efforts, but the WHO now draws in part on the GBD methodology when producing its global health estimates. ${ }^{39}$ However, modelled GBD study estimates are reviewed by international collaborators, and estimates that are inconsistent with observed data or not aligned with expected results are further examined.

\section{Conclusion}

Our study, with its synthesis of multiple countrywide data sources, provides a comprehensive Canadian analysis of both disability and premature death on the same scale in relation to risk factors and delivers an unprecedented assessment and ranking of the risk factors attributable to health loss in Canada over time. Modifiable metabolic and behavioural risk factors such as tobacco and diet have remained the leading risk factors in Canada for the last 27 years, findings that show opportunities for reducing Canada's health burden. These findings persist despite some substantial declines in risk factor exposure, showing that risk factors can influence the population for a substantial length of time and that decreasing health burden for Canadians requires a long-term commitment to risk reduction. Future analyses are warranted that focus on specific risk factors, detailing their complexities. This paper is part of an ongoing assessment of the burden of disease attributable to risk factors in Canada, with each iteration benefitting from newly collected data. GBD estimates provide unique insight into the relative magnitude of the contribution of many important risk factors to health loss and can guide decision-makers, clinicians and scientists as they identify priorities and targets for reducing the burden of premature death and disability in Canada.

\section{References}

1. Manuel DG, Perez R, Sanmartin C, et al. Measuring burden of unhealthy behaviours using a multivariable predictive approach: life expectancy lost in Canada attributable to smoking, alcohol, physical inactivity, and diet. PLoS Med 2016;13:e1002082.

2. Global Burden of Disease Study Collaborators. Global, regional, and national age-sex specific all-cause and cause-specific mortality for 240 causes of death, 1990-2013: a systematic analysis for the Global Burden of Disease Study 2013. Lancet 2015;385:117-71.

3. Smith JN. Epic measures: One doctor. Seven billion patients. 1st ed. New York: HarperCollins; 2015.

4. Murray CJ, Atkinson C, Bhalla K, et al. The state of US health, 1990-2010: burden of diseases, injuries, and risk factors. FAMA 2013;310:591-608.

5. Mokdad AH, Forouzanfar MH, Daoud F, et al. Global burden of diseases, injuries, and risk factors for young people's health during 1990-2013: a systematic analysis for the Global Burden of Disease Study 2013. Lancet 2016;387:2383-401.

6. Plass D, Vos T, Hornberg C, et al. Trends in disease burden in Germany: results, implications and limitations of the Global Burden of Disease study. Dtsch Arztebl Int 2014;111:629-38.

7. Murray CJ, Richards MA, Newton JN, et al. UK health performance: findings of the Global Burden of Disease Study 2010. Lancet 2013;381:997-1020.

8. Lang JJ, Alam S, Cahill LE, et al. Global Burden of Disease Study trends for Canada from 1990 to 2016. CMA7 2018;190:E1296-304

9. Murray CJL, Lopez AD. Measuring global health: motivation and evolution of the Global Burden of Disease Study. Lancet 2017;390:1460-4.

10. Global Burden of Disease Study Collaborators. Global, regional, and national comparative risk assessment of 79 behavioural, environmental and occupational, and metabolic risks or clusters of risks, 1990-2015: a systematic analysis for the Global Burden of Disease Study 2015. Lancet 2016;388:1659-724.

11. GBD 2016 Mortality Collaborators. Global, regional, and national under-5 mortality, adult mortality, age-specific mortality, and life expectancy, 19702016: a systematic analysis for the Global Burden of Disease Study 2016. Lancet 2017;390:1084-150.

12. Global Burden of Disease Study Collaborators. Global, regional, and national disability-adjusted life-years (DALYs) for 333 diseases and injuries and healthy life expectancy (HALE) for 195 countries and territories, 1990-2016: a systematic analysis for the Global Burden of Disease Study 2016. Lancet 2017;390: 1260-344.

13. Global Burden of Disease Study Collaborators. Global, regional, and national incidence, prevalence, and years lived with disability for 328 diseases and injuries for 195 countries, 1990-2016: a systematic analysis for the Global Burden of Disease Study 2016. Lancet 2017;390:1211-59.

14. Global Burden of Disease Study Collaborators. Global, regional, and national under-5 mortality, adult mortality, age-specific mortality, and life expectancy, 1970-2016: a systematic analysis for the Global Burden of Disease Study 2016. Lancet 2017;390:1084-150.

15. Global Burden of Disease Study Collaborators. Global, regional, and national comparative risk assessment of 84 behavioural, environmental and occupational, and metabolic risks or clusters of risks, 1990-2016: a systematic analysis for the Global Burden of Disease Study 2016. Lancet 2017;390:1345-422.

16. Global Burden of Disease Study Collaborators. Measuring progress and projecting attainment on the basis of past trends of the health-related Sustainable Development Goals in 188 countries: an analysis from the Global Burden of Disease Study 2016. Lancet 2017;390:1423-59.

17. GBD Compare Data Visualization. Seattle (WA): Institute of Health Metrics and Evaluation (IHME); 2016. Available: http://vizhub.healthdata.org/ gbd-compare (accessed 2017 Oct. 30)

18. Stevens GA, Alkema L, Black RE, et al. Guidelines for Accurate and Transparent Health Estimates Reporting: the GATHER statement. Lancet 2016;388: e19-23.

19. Global Burden of Disease Study Collaborators. Global, regional, and national incidence, prevalence, and years lived with disability for 301 acute and chronic diseases and injuries in 188 countries, 1990-2013: a systematic analysis for the Global Burden of Disease Study 2013. Lancet 2015;386:743-800.

20. Haagsma JA, Maertens de Noordhout C, Polinder S, et al. Assessing disability weights based on the responses of 30660 people from four European countries. Popul Health Metr 2015;13:10

21. Salomon JA, Haagsma JA, Davis A, et al. Disability weights for the Global Burden of Disease 2013 study. Lancet Glob Health 2015;3:e712-23.

22. Murray CJ, Lopez AD. On the comparable quantification of health risks: lessons from the Global Burden of Disease study. Epidemiology 1999;10:594-605.

23. Gupta PD. Standardization and decomposition of rates: a user's manual. Washington (DC): US Department of Commerce, Economics and Statistics Administration, Bureau of the Census; 1993.

24. Shibuya K, Inoue M, Lopez AD. Statistical modeling and projections of lung cancer mortality in 4 industrialized countries. Int 7 Cancer 2005;117:476-85.

25. Jaakkola MS, Samet JM. Occupational exposure to environmental tobacco smoke and health risk assessment. Environ Health Perspect 1999;107(Suppl 6):829-35.

26. Currie SR, Nesbitt K, Wood C, et al. Survey of smoking cessation services in Canadian addiction programs. 7 Subst Abuse Treat 2003;24:59-65.

27. Steensma C, Loukine L, Orpana H, et al. Comparing life expectancy and health-adjusted life expectancy by body mass index category in adult Canadians: a descriptive study. Popul Health Metr 2013;11:21.

28. Rehm J, Patra J, Popova S. Alcohol-attributable mortality and potential years of life lost in Canada 2001: implications for prevention and policy. Addiction 2006;101:373-84.

29. Shield KD, Taylor B, Kehoe T, et al. Mortality and potential years of life lost attributable to alcohol consumption in Canada in 2005. BMC Public Health 2012;12:91.

30. Williams DR, Priest N, Anderson NB. Understanding associations among race, socioeconomic status, and health: patterns and prospects. Health Psychol 2016;35:407-11

31. Kyu HH, Georgiades K, MacMillan HL, et al. Community- and individuallevel factors associated with smoking and heavy drinking among Aboriginal people in Canada. Can 7 Public Health 2015;106:e22-8.

32. Williams DR, Mohammed SA, Leavell J, et al. Race, socioeconomic status, and health: complexities, ongoing challenges, and research opportunities. Ann NY Acad Sci 2010;1186:69-101.

33. Manuel DG, Leung M, Nguyen K, Canadian Cardiovascular Outcomes Research Team; et al. Burden of cardiovascular disease in Canada. Can 7 Cardiol 2003;19:997-1004.

34. Prus SG. Comparing social determinants of self-rated health across the United States and Canada. Soc Sci Med 2011;73:50-9.

35. Lee DS, Chiu M, Manuel DG, et al. Trends in risk factors for cardiovascular disease in Canada: temporal, socio-demographic and geographic factors. CMA7 2009; 181:E55-66.

36. Diabetes Prevention Program Research Group; Knowler WC, Fowler SE, Hammon RF, et al. 10-year follow-up of diabetes incidence and weight loss in the Diabetes Prevention Program Outcomes Study. Lancet 2009;374: 1677-86.

37. Holt-Lunstad J, Smith TB, Baker M, et al. Loneliness and social isolation as risk factors for mortality: a meta-analytic review. Perspect Psychol Sci 2015;10:227-37.

38. Valtorta NK, Kanaan M, Gilbody S, et al. Loneliness and social isolation as risk factors for coronary heart disease and stroke: systematic review and metaanalysis of longitudinal observational studies. Heart 2016;102:1009-16. 
39. WHO methods and data sources for global burden of disease estimates 20002016. Geneva: World Health Organization, Department of Information, Evidence and Research; 2018.

Affiliations: Departments of Medicine (Alam, Cahill) and of Community Health and Epidemiology (Alam, Cahill), Dalhousie University, Halifax, NS; Healthy Active Living and Obesity Research Group (Lang), CHEO Research Institute, Ottawa, Ont.; Centre for Surveillance and Applied Research (Lang, Orpana), Health Promotion and Chronic Disease Prevention Branch, Public Health Agency of Canada, Ottawa, Ont.; Division of Dermatology (Drucker), Department of Medicine, and Women's College Research Institute, Women's College Hospital, Toronto, Ont.; Division of Dermatology (Drucker), Department of Medicine, University of Toronto, Toronto, Ont.; Centre of Excellence in Cancer Prevention (Gotay), School of Population and Public Health, Faculty of Medicine, University of British Columbia, Vancouver, BC; Centre for Addiction and Mental Health (Kozloff), Toronto, Ont.; Department of Psychiatry (Kozloff), University of Toronto, Toronto, Ont.; School of Physical and Occupational Therapy (Mate), McGill University, Montréal, Que.; Departments of Community Health Sciences and Psychiatry (Patten), University of Calgary, Calgary, Alta.; School of Epidemiology and Public Health (Orpana),
University of Ottawa, Ottawa, Ont.; Institute for Health Metrics and Evaluation (Afshin), Seattle, Wash.; Department of Nutrition (Cahill), Harvard T.H. Chan School of Public Health, Boston, Mass.

Contributors: All authors contributed to conceiving the research questions and objectives and interpreting the results. Samiah Alam and Leah Cahill drafted the manuscript. All authors revised the manuscript for important intellectual content. All authors approved the final manuscript and agreed to be accountable for all aspects of the work.

Funding: This research was conducted as part of the Global Burden of Diseases, Injuries, and Risk Factors Study, coordinated by the Institute for Health Metrics and Evaluation. The Global Burden of Disease Study was partially funded by the Bill \& Melinda Gates Foundation; the funders had no role in the study design, data analysis, data interpretation or writing of the report. Samiah Alam was the recipient of an experiential learning fund through the Direct Skills Link program with the Department of Labour and Advanced Education, Government of Nova Scotia, Canada.

Supplemental information: For reviewer comments and the original submission of this manuscript, please see www.cmajopen.ca/content/7/1/ E140/suppl/DC1. 